\title{
Stress analysis of a second stage gas turbine blade under asymmetric thermal gradient
}

\author{
Javad Rahimi, Esmaeil Poursaeidi, and Ehsan Khavasi* \\ Mechanical Engineering Department, University of Zanjan, Zanjan, Zanjan Province, Iran
}

Received: 1 July 2018 / Accepted: 11 July 2019

\begin{abstract}
In this study the main causes of the failure of a GE-F9 second stage turbine blade were investigated. The stress distribution of the blade which has 6 cooling vents in three modes (with full cooling, closure of half of the cooling channels, and without cooling) was studied. A three dimensional model of the blade was built and the fluid flow on the blade was studied using the FVM method. The stress distribution due to centrifugal forces applied to the blade, temperature gradients and aerodynamic forces on the blade surface was calculated by the finite element model. The results show that the highest temperature gradient and as a result the highest stress value occurs for the semi-cooling state at the areas near the blade root and this status is true for the full cooling mode for the regions far from the root. However, the field observations showed that the failure occurred for the blade with the semi-cooling state (due to closure of some of the channels) at areas far from the root. It is discussed that the main factor of the failure is not the stress values being maximum because in the state of full cooling mode (the state with the maximum stress values) the temperature of the blade is the lowest state and as a result the material properties of the blade show a better resistance to phenomena like hot corrosion and creep.
\end{abstract}

Keywords: turbine blade failure / blade cooling / thermal stress / stress analysis / finite element modelling

\section{Introduction}

One of the important components of gas turbines is their moving blades which are under mechanical and thermal stresses due to high-speed rotation and exposure to high temperatures. To improve turbine efficiency, gas turbine inlet temperature should be increased $[1,2]$. On the other hand, the temperature of the turbine blades needs to be kept lower than a certain value because of the limitations of the material properties at high temperatures. In order to achieve ideal conditions in the design and manufacture of gas blades, the accuracy in the measurement of the temperature distribution of the blade is very important. So, in recent years, many studies have been done to estimate temperature and stress distribution of the turbine blade [3], turbulence intensity of the streamline [4,5], the Reynolds number and Mach number [6,7], to experimentally study the effect of cooling temperatures and mass flow on the heat transfer distribution on the turbine blades [8], swirl effects of unsteady vortices $[9,10]$ as well as the tip and shape of the blade $[11,12]$. In addition to these experiments, numerical studies have been carried out using CFD codes developed based on the Navier-Stokes equations and boundary layer

\footnotetext{
* e-mail: khavasi@znu.ac.ir
}

models. Among numerical studies done, we can note the physical effect of the flow in the cooling hole on the heat transfer in turbine blade [13], improvement and development of turbulence models, in order to accurately predict heat transfer from the surface of the turbine blade with cooling and without cooling [14]. However, most of the numerical simulations conducted in this matter are 2 dimensional or does not applied on near-real conditions. So a thorough assessment considering the real geometry and near-real boundary conditions needs to be done.

In this study, numerical analysis is conducted in order to estimate thermal stresses due to temperature gradient and stresses caused by aerodynamic and centrifugal effects on a fractured blade.

Figure 1 shows the fractured blade, as it can be seen from this illustration.

\section{Geometry, boundary conditions and material properties}

The model of blade was designed using CAD software. Since there are several effective parameters such as complex geometry of the blade, the unsteady nature of the flow, relative motion of the components and turbulence in order to obtain accurate results, there is a need for proper 


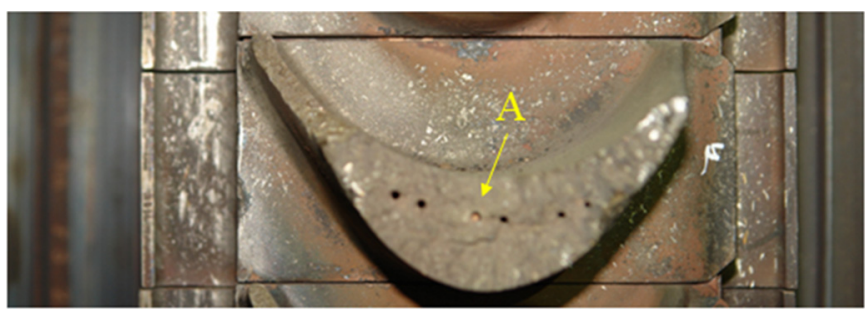

Fig. 1. Broken blades of the second stage of power plant and obstruction of one of the cooling channels.

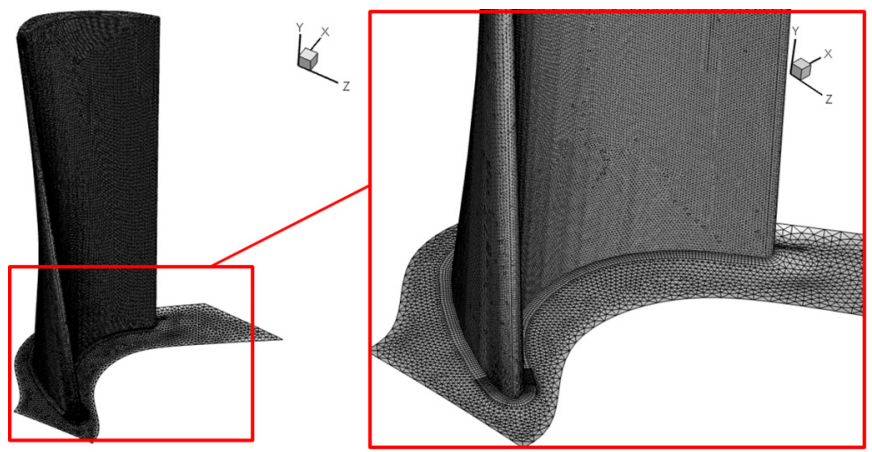

Fig. 2. GEF9 turbine blade meshing using boundary layer mesh.

meshing. For this purpose, several meshing with different numbers of elements was built by Gambit 2.4.6 software and mesh independency was investigated. In order to analyze the fluid flow, the sector volume around the blade was calculated considering the arrangement of 93 adjacent turbine blades of the second stage. A total of 3,287,137 structured elements were used, 1,482,314 elements of which are for the blade body (Fig. 2), and the rest for the environment around the blade and 6 cooling holes (Fig. 3). It should be noted that according to Figure 2 a boundary layer mesh was used in areas near surface of the blade.

Boundary conditions used in this study is based on measurements done in the power plant. According to Figure 4 the inlet mass flow rate is $4 \mathrm{~kg} / \mathrm{s}$ with the temperature of $1149^{\circ} \mathrm{K}$. In the case of cooling channels, the inlet cooling fluid mass flow rate from the compressor is $7.2 \times 10^{-4} \mathrm{~kg} / \mathrm{s}$ for each channel, and its temperature is 620 Kelvin. Periodic boundary condition is used for the lateral boundaries; the output pressure boundary condition is used for the outlet surface.

Since the flow around the turbine blade is turbulent, $k$ $-\varepsilon$ model was used to simulate the flow. The convergence criteria determined to be $10^{-6}$ for the residuals.

The material properties of the GTD 111 nickel-base alloy are shown in Table 1.

\section{Results and discussion}

In the sight of theory, the thermal stresses, static and dynamic forces that affect the performance of the turbine blades are as follows:

- the aerodynamic forces of flow;

- the stresses resulting from temperature gradient;

- forces due to centrifugal effect.

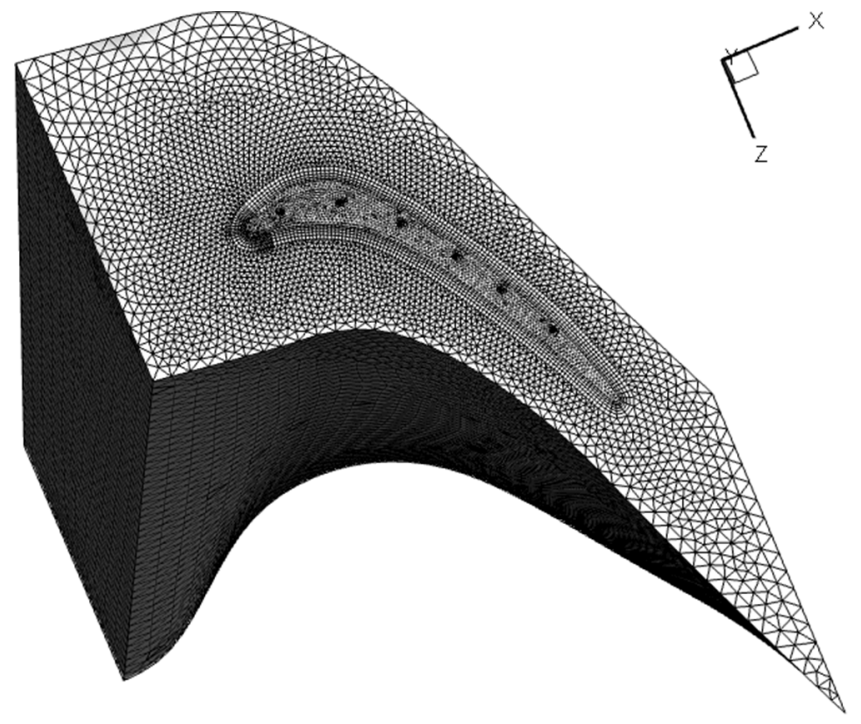

Fig. 3. Mesh the surrounding environment of the blade and cooling holes.

In this part these that the effect of each parameter on the blade performance will be explained.

\subsection{Fluid flow analysis}

The uniform distribution of the force from the fluid pressure from bottom to top of the blade is of great importance. Unequal distribution of the force makes the gases flow over the blade with different speed and pressure. The difference in rotational speed at the hub and tip of the blade reduces the gases relative speed at the tip, so less force is applied to the tip of the blade compared with hub. So, modern gas turbines use blades that have impaction at the hub and reaction at the tip.

Figure 5 shows the distribution of static pressure on the blade. Pressure drop required for the blade reaction appears at the tip, and gradually changes to the conditions without drop for the impaction at the hub. High pressure at the tip makes the gases move toward the roots of the blade; this effect is opposed to centrifugal forces which flow gases into the tip. As a result, a uniform force distribution occurs across the entire blade.

Figure 6 shows the static pressure coefficient which is obtained by $C_{p}=\left[P_{x}-P_{\text {ref }}\right] /\left[1 / 2 p V_{b}^{2}\right]$, where $P_{x}$ and $P_{\text {ref }}$ are the static pressure on the mid-surface of the blade and the reference pressure on the stagnation point, respectively. $V_{b}$ is the speed at the bladeinput that is used as a reference for dynamic pressure term. The difference of the results from the present study and the result from reference [3] is due to the physical conditions of the flow.

\subsection{Heat transfer analysis}

Figure 7 shows the comparison of the heat transfer coefficient in the middle part of the blade in the recent study and the amount of mass and heat transfer coefficient in the previous studies conducted by others $[3,4,5,9,10]$. 


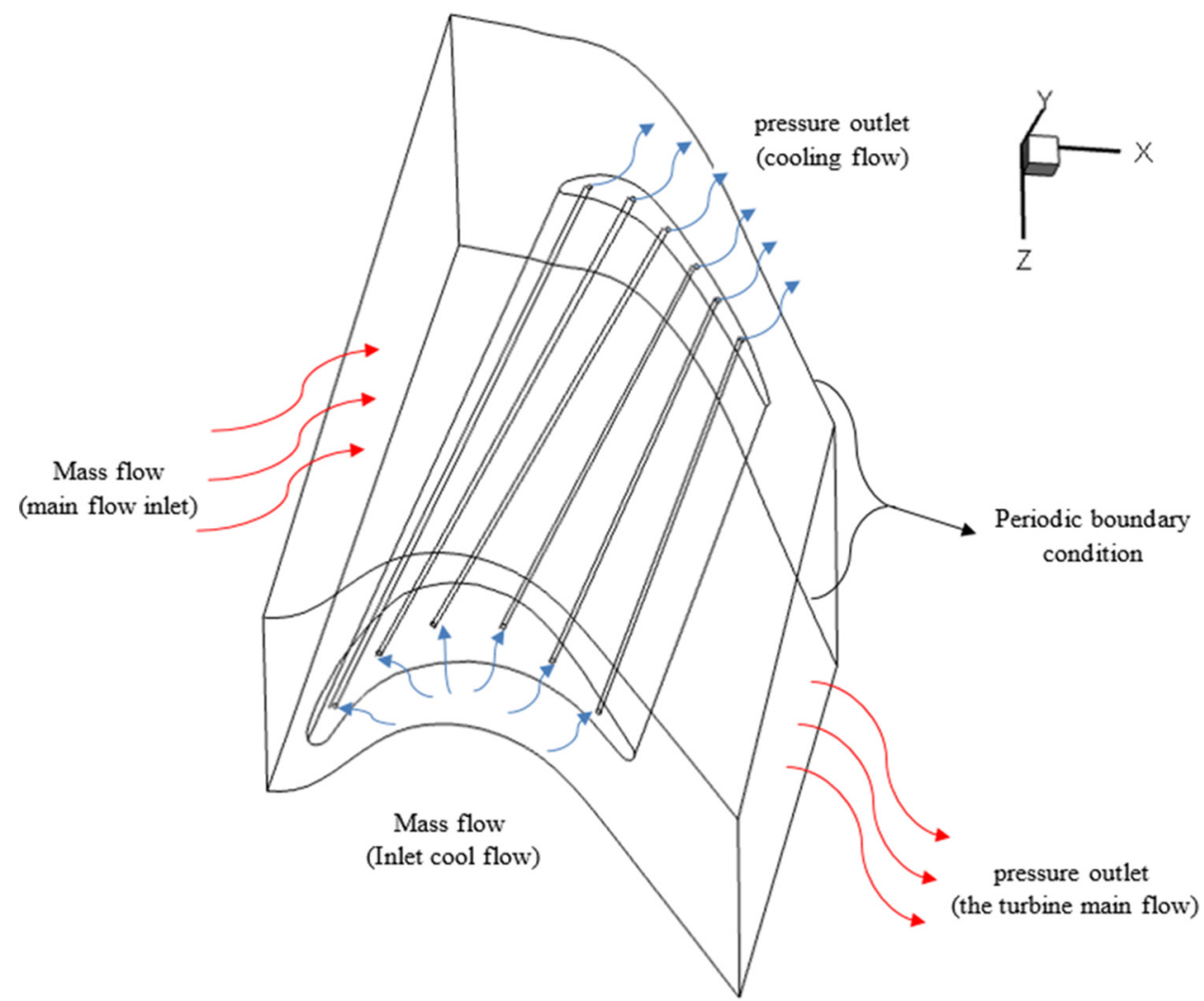

Fig. 4. Boundary conditions of the flow variables with 6 cooling channels.

Table 1. Properties of the alloy GTD-111 [15].

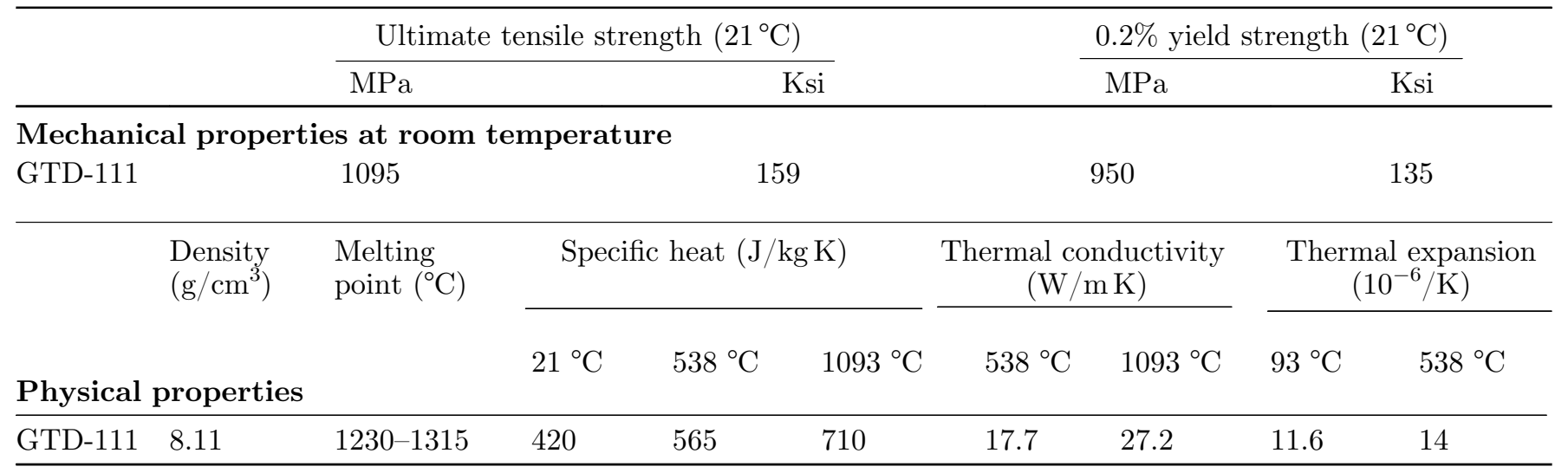

The highest amount of heat transfer occurs due to collision of the main flow on the stagnation point at leading edge. The lowest amount of heat transfer coefficient occurs due to the development of thermal boundary layer at the trailing edge. The highest temperature is observed at the blade tip.

In this study, the Reynolds number is considered to be equal to $1.07 \times 10^{6}$. One of the reasons for the different results in some parts is the difference in the blade geometry studied by other researchers. Of course, another thing that has impact on the change of these results is the condition in which the flow passes the blade surface.

Since the effect of temperature gradient on stress distribution occurring in the blade is much more than the pressure of the fluid on the blade, and with respect to blades' working conditions, there is blocking probability in some cooling channels, therefore, for better comparison, simulation is done in three modes of (1) full cooling with 6 cooling channels; (2) cooling assuming the blockage of half of the cooling channels (Fig. 8); (3) assuming the blockage of all cooling channels. 
In order to find the temperature distribution and Stress caused by temperature gradients in the blade, 4 sections are considered with similar distances in the blade according to Figure 9 for all three cooling modes.

Figure 10 shows the temperature distribution and stress caused by temperature gradients in section 1 for all three modes. In the full cooling with 6 cooling channels, maximum blade temperature is significantly lower than the other two conditions. The highest temperature gradient is in the half-cooling mode and is equal to 74 Kelvin. The maximum stresses caused by the temperature gradient in this section is related to half-cooling state.

Figure 11 shows the section 2 at the distance of one third of the blades' length from hub of the blade. With increasing altitude, average temperature is increased in all
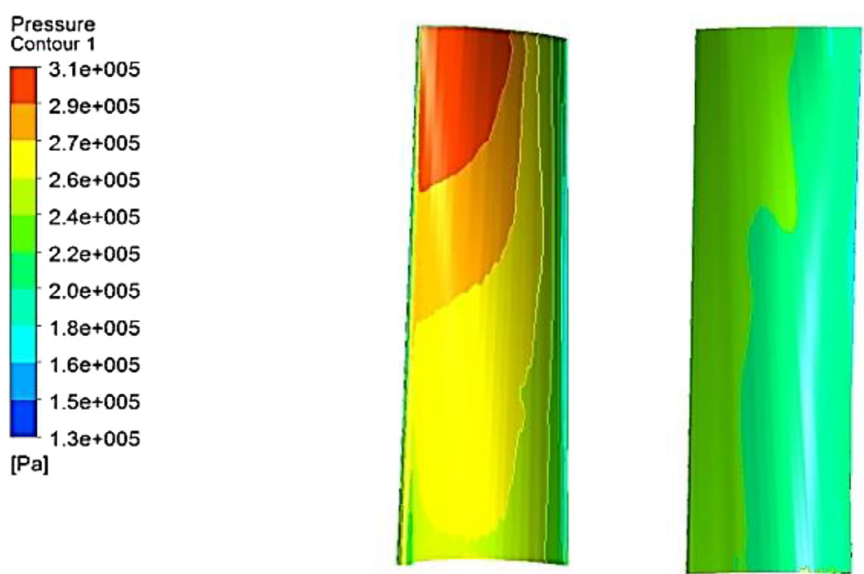

Fig. 5. Distribution of pressure on both sides of the blade. three modes but the temperature gradient is reduced at any section. On the other hand with reduces the temperature gradient we can also see decrease of corresponding stresses.

Figure 12 shows the section 3 at the distance of two third of the blades' length from hub of the blade. The blade without cooling is subjected to relatively little temperature changes. In blades with cooling channels, as height increases the cooling fluid impact reduces, so the section temperature will be increased. The maximum temperature of the section (3) happens at the trailing edge of the blade in all three modes, and the highest temperature is in the mode without cooling. In this section also a reduction in the stresses caused by the temperature gradient in three sections can be seen.

Figure 13 shows near the blade tip, i.e. section (4). Compared to all sections, the maximum temperature occurred in this section, and in all cases, the trailing edge of the blade is in high temperature which is due to the cooling fluid warming and its low impact in this height of the blades. On the other hand, the temperature difference between the three modes is reduced. On the other hand the thermal stress at this section is the lowest and is near together in three states.

Figure 14 shows the temperature distribution on the wall attached to the cooling fluid with 6 cooling channels. The results show that with increasing altitude, cooling fluid gets warmer and the wall temperature goes higher.

\subsection{Centrifugal force}

This force is a function of the rotor rotation speed, turning radius and mass of blades. This force is a harmful one for the blades so, designers are always trying to reduce mass of blades to minimize its amount. In order to calculate
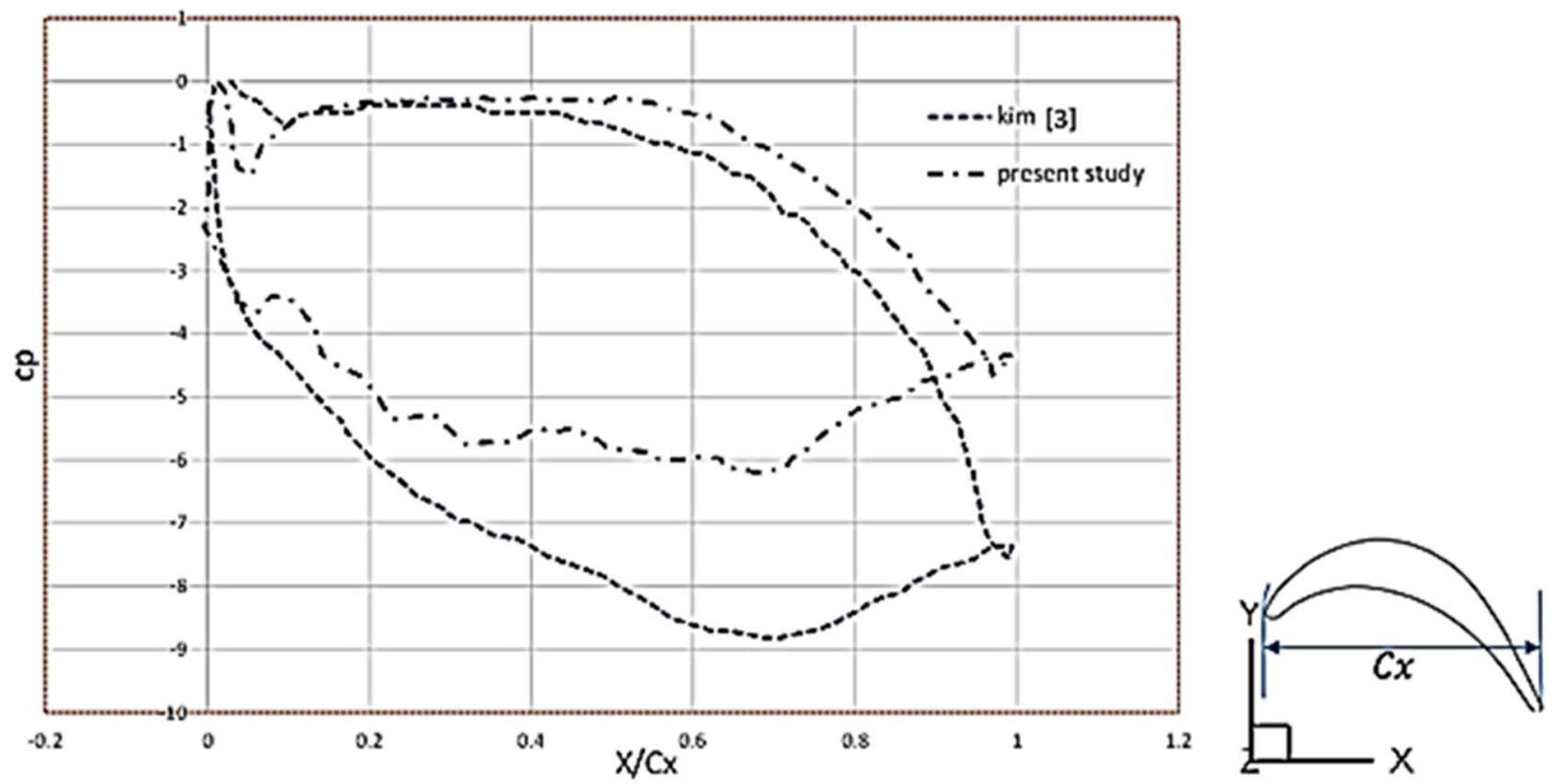

Fig. 6. Static pressure coefficient on the mid-surface of the blade. 


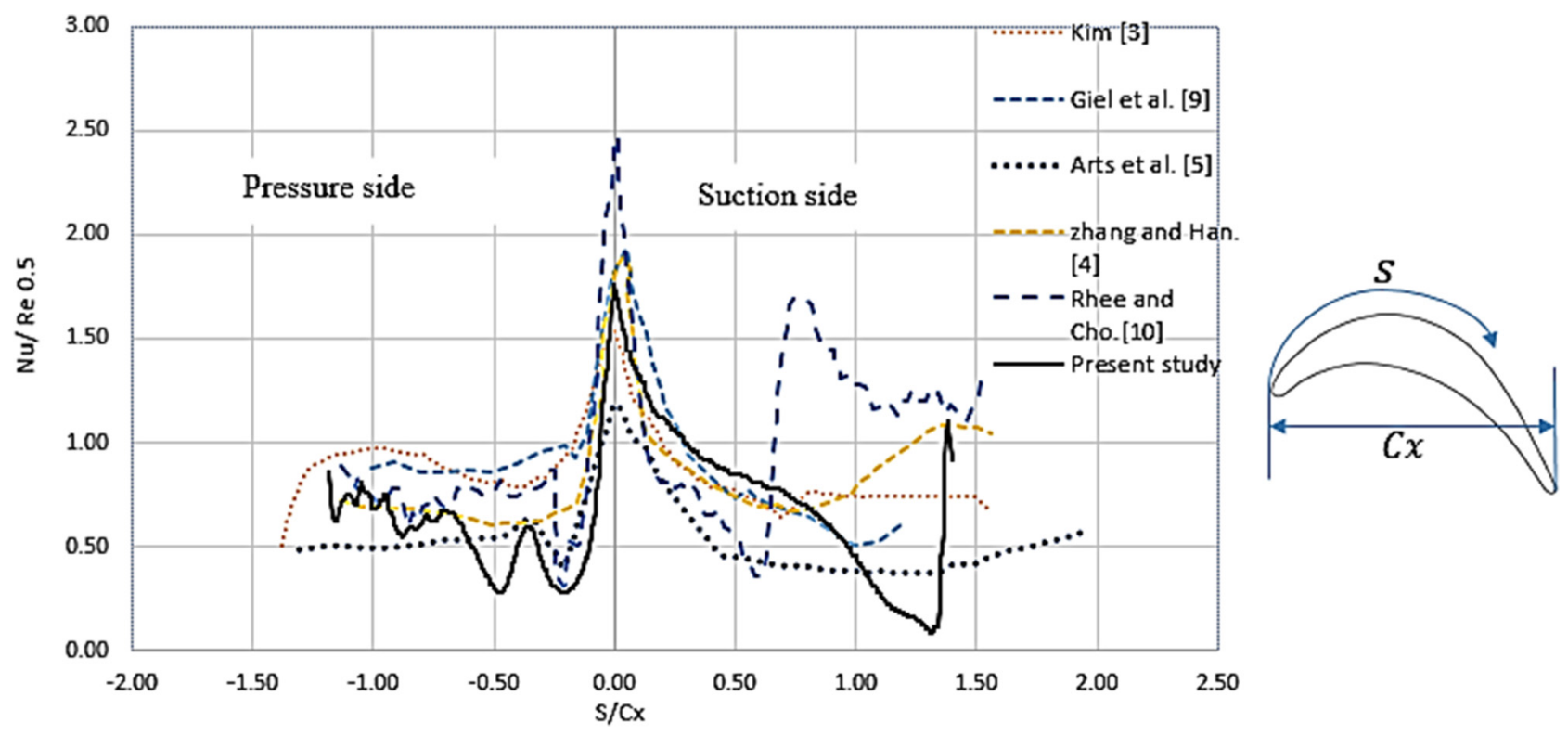

Fig. 7. Comparing the local heat transfer on the middle part of the blade in previous researches with the current study.

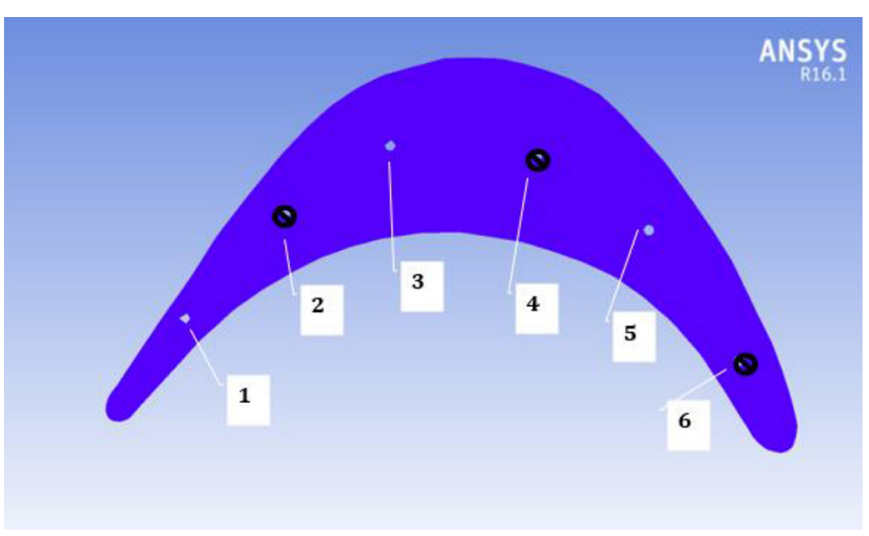

Fig. 8. Cooling channels in semi-open mode $(2,4$ and 6 are closed).

analytically the function of the changes in stress distribution caused by the centrifugal force along a blade with variable cross-section, we act as follows [16]:

$$
\begin{gathered}
\sigma_{r}=F_{r} / S_{r} \\
F_{r}=F_{b l(r)}+F_{b}
\end{gathered}
$$

where $F_{r}$ is the amount of centrifugal force and $S_{r}$ is the area function at any section of the blade, both of which are defined as functions of the turning radius of blades. According to Figure $15, R_{\mathrm{hub}}=$ the radius of the blade hub and $R_{\mathrm{tip}}=$ the radius of the blade tip. In relation $2, F_{b}$ parameter is the centrifugal force of the Shroud Banding which is considered to be zero for this kind of blade due to the lack of Shroud Banding part. Centrifugal force of $F_{b l(r)}$

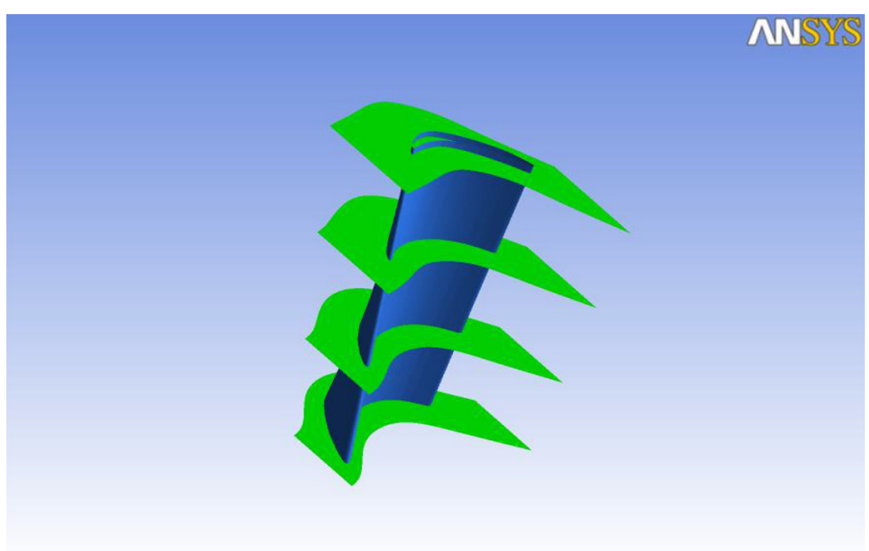

Fig. 9. Plotted sections for blade temperature analysis.

is a piece of the blade, which is applied in the area between section $r$ and blade end radius $R_{\text {tip }}$ with rotational speed of $3000 \mathrm{rpm}$. In order to calculate the $F_{b l}$ for the airfoil, according to Figure 15, considering a longitudinal element, centrifugal force is achieved.

$$
F_{b l(r)}=\int_{r-R_{\mathrm{Hub}}}^{l} \rho \omega^{2} S_{(\xi)}\left(R_{\mathrm{Hub}}+\xi\right) d \xi .
$$

To compute the integral in equation (3) area function $\left(S_{(\xi)}\right)$ needs to be obtained. To evaluate $S_{(\xi)}$ the area of forty different sections of blade obtained and interpolated. $S_{(\xi)}$ is achieved as follow:

$$
\begin{aligned}
S_{(\xi)}= & -3.02 \times 10^{-5} \xi^{3}+1.36 \times 10^{-2} \xi^{2}-9.9 \times 10^{-3} \xi \\
& +2.58 \times 10^{-3} .
\end{aligned}
$$



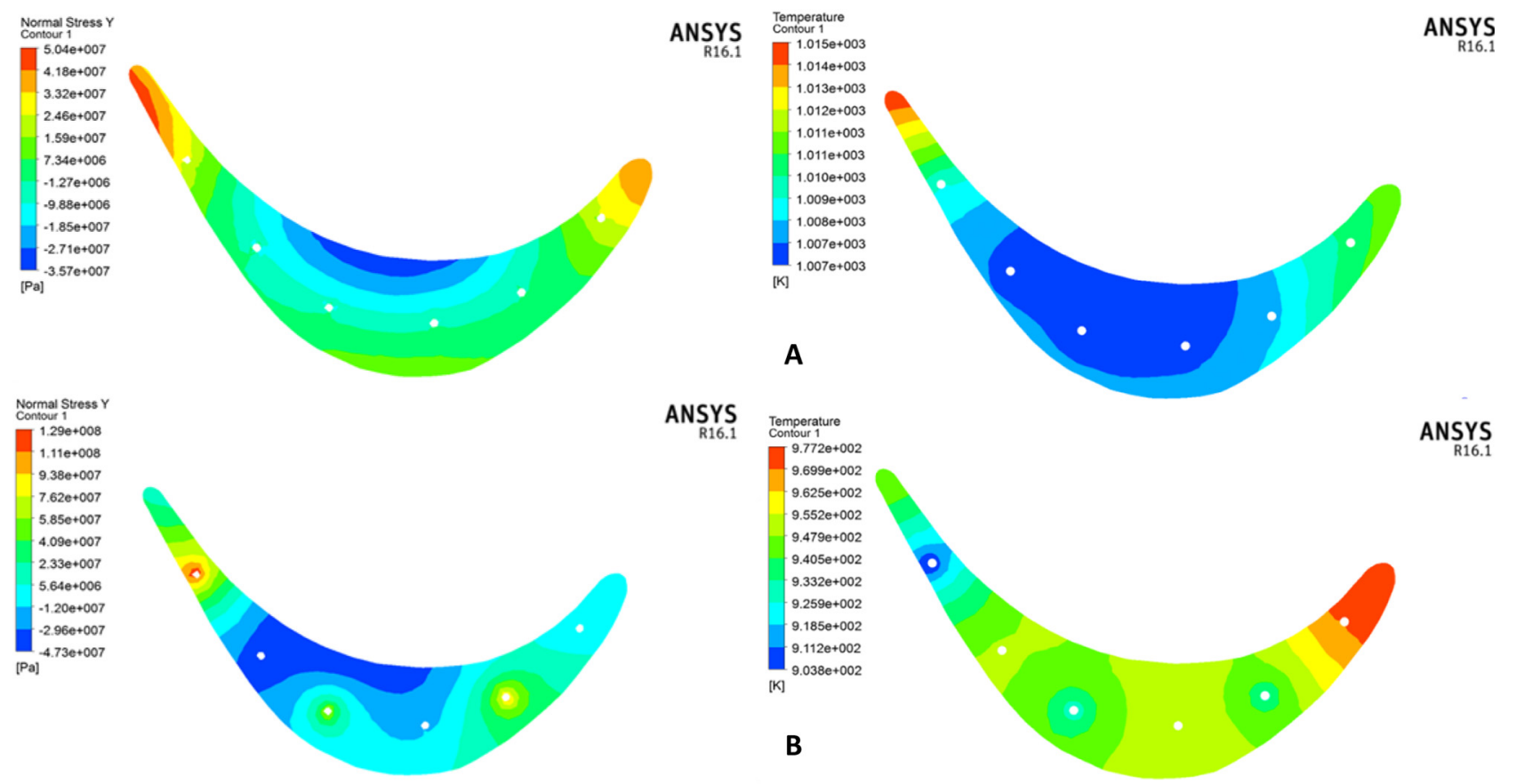

ANSYS
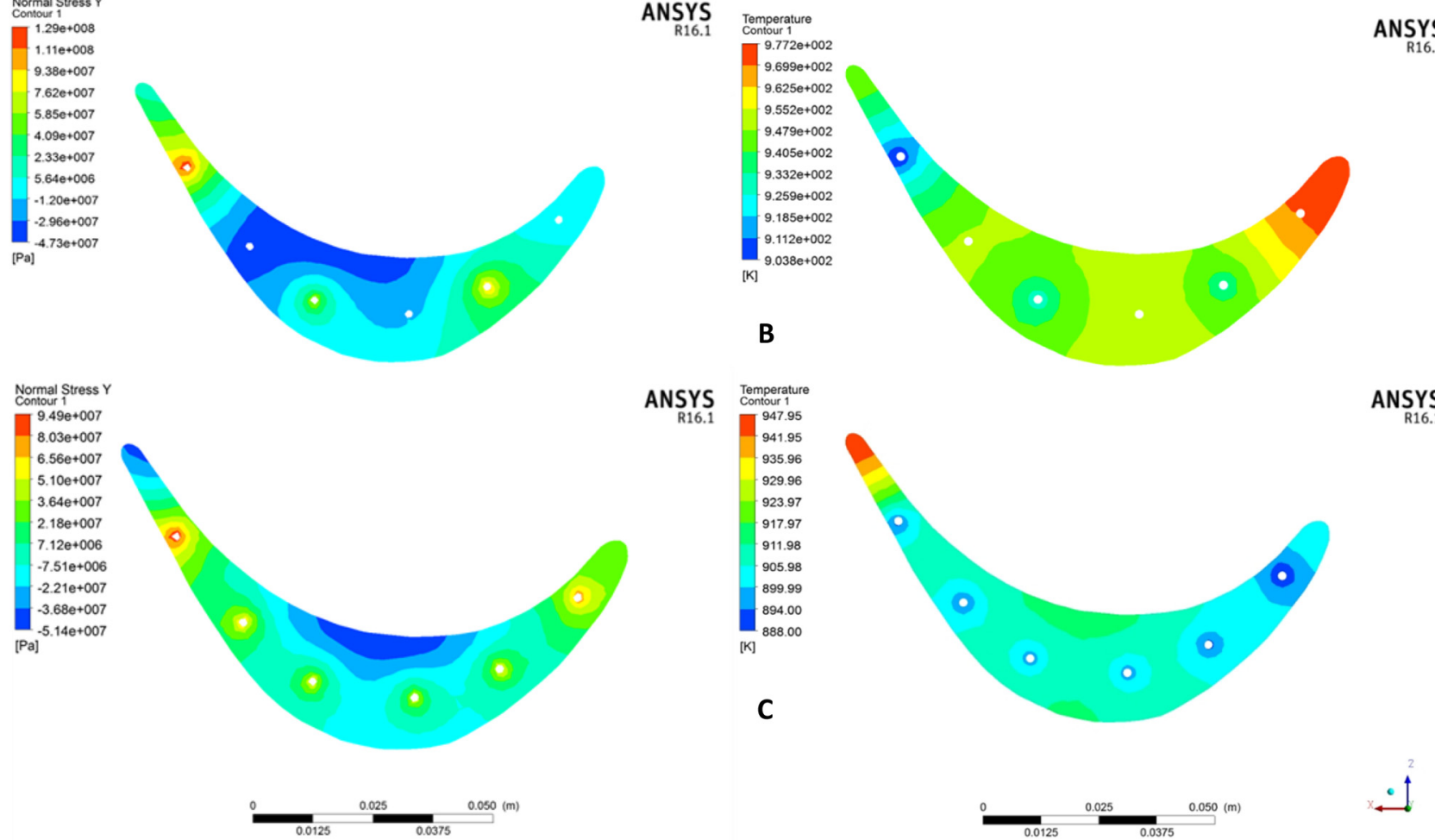

Fig. 10. Temperature distribution and normal stress caused by temperature gradients in section (1) near the hub in the modes (a) without cooling, (b) half-cooling, (c) full cooling.

Consequently $F_{r}$ can be obtained:

$$
\begin{aligned}
F_{r}= & -4.837 \times 10^{3} r^{5}+2.730 \times 10^{6} r^{4}+9.34 \times 10^{5} r^{3} \\
& -2.851 \times 10^{6} r^{2}+2.022 \times 10^{6} r .
\end{aligned}
$$

Finally, having the area and force functions, the stress distribution function can be obtained in accordance with equation (1) which is shown by solid line in Figure 16. To ensure the validity of the results obtained by numerical simulation, the stress distribution due to centrifugal force compared by analytical results. Numerical results (the average stress in each section) are also illustrated by circles in this figure. The agreement between the results is suitable and the differences can be because of assumptions have been made in the analytical method; normal stress distribution in each section is assumed to be constant and the deformation of the blade is ignored.
To examine the stress on the blade from the fluid, the results of the fluid analysis, including temperature and pressure distribution on the blade, are inserted as boundary conditions; and the stresses obtained in different sections of the blade are investigated by applying a $3000 \mathrm{rpm}$ rotation on the blade Figure 17 shows the overall stress that applied on the blades in half cooling state.

Field surveys of the second stage blade fracture surfaces show that the fractures occurred at the section 2 and lower sections of the blade, started to grow and finally caused the failure of the blade. Thermo-mechanical analyses also show the fact that the maximum stress occurred in the hub. It seems this is because of high temperature gradients in this area due to the proximity of these pages to the inlet opening of the cooling fluid on the one hand, and inability of deformation as a result of root constraint, on the other hand. 


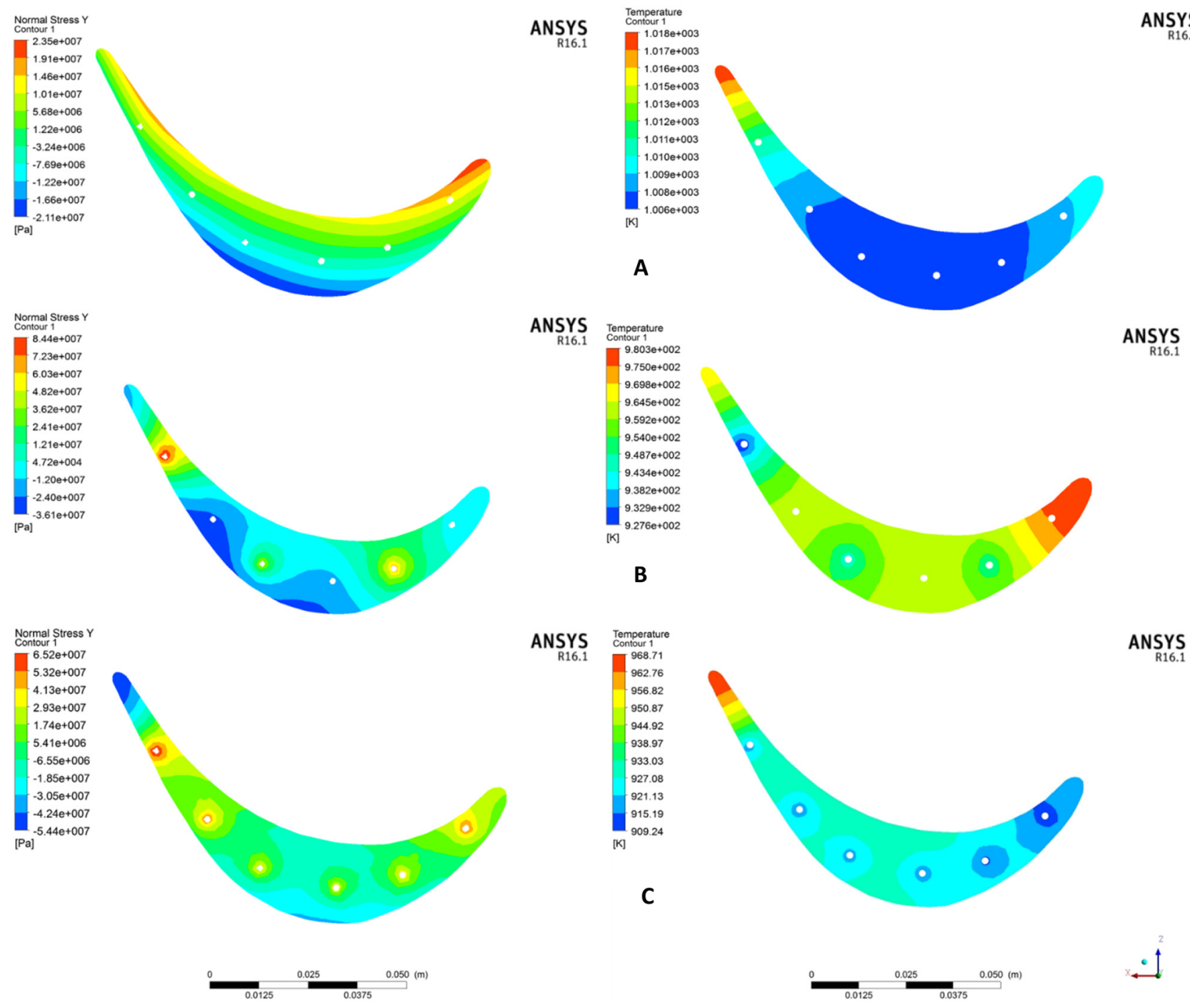

Fig. 11. Temperature distribution and normal stress caused by temperature gradients in section (2) at the distance of one third of the blades' length from hub of the blade in the modes (a) without cooling, (b) half-cooling, (c) full cooling.

\section{Conclusion}

Internal cooling of GEF9 turbine blade was presented and investigated in this paper. In the first step, the cooling air flow inside the channel was simulated and evaluated. In the next step, normal stresses were evaluated in four sections using the results obtained from the fluid analyses and cooling system effect on the temperature parameters. According to the results presented in this report, the following cases can be presented as the conclusion:

- Using cooling system as a channel in the blade significantly reduces the temperature in all parts of the blade. Results showed that maximum temperature is in the mode without cooling at all sections, and the temperature difference is more tangible in the modes of cooling and without cooling in the blade root, and this difference decreases with increasing altitude; but in general, the temperature increases with increased blade height, and maximum temperature occurs in the upper part of the blade.
- It can be said about the pressure that in three modes of 6- and 3-channel cooling and without cooling, there is no significant change in the pressure on the blades, and the pressure is almost equal in all modes, and the highest pressure is at the stagnation point near the peak leading. Another important point is that the influence of this parameter is very low compared to the impact of stresses caused by rotation and temperature gradient.

- Investigating the four sections cut on the blade, it can be concluded about the applied stress that the stress will be reduced gradually with increasing distance from the blade root, and the maximum stress occurs in the section near the blade root. These stresses are caused by applying blade rotation, temperature gradient and fluid pressure on the blade. Comparing the three modes above, it can be found that highest stress is related to the 6-channel cooling mode. Stress difference between cooling and without cooling modes is at maximum at the blade root. 

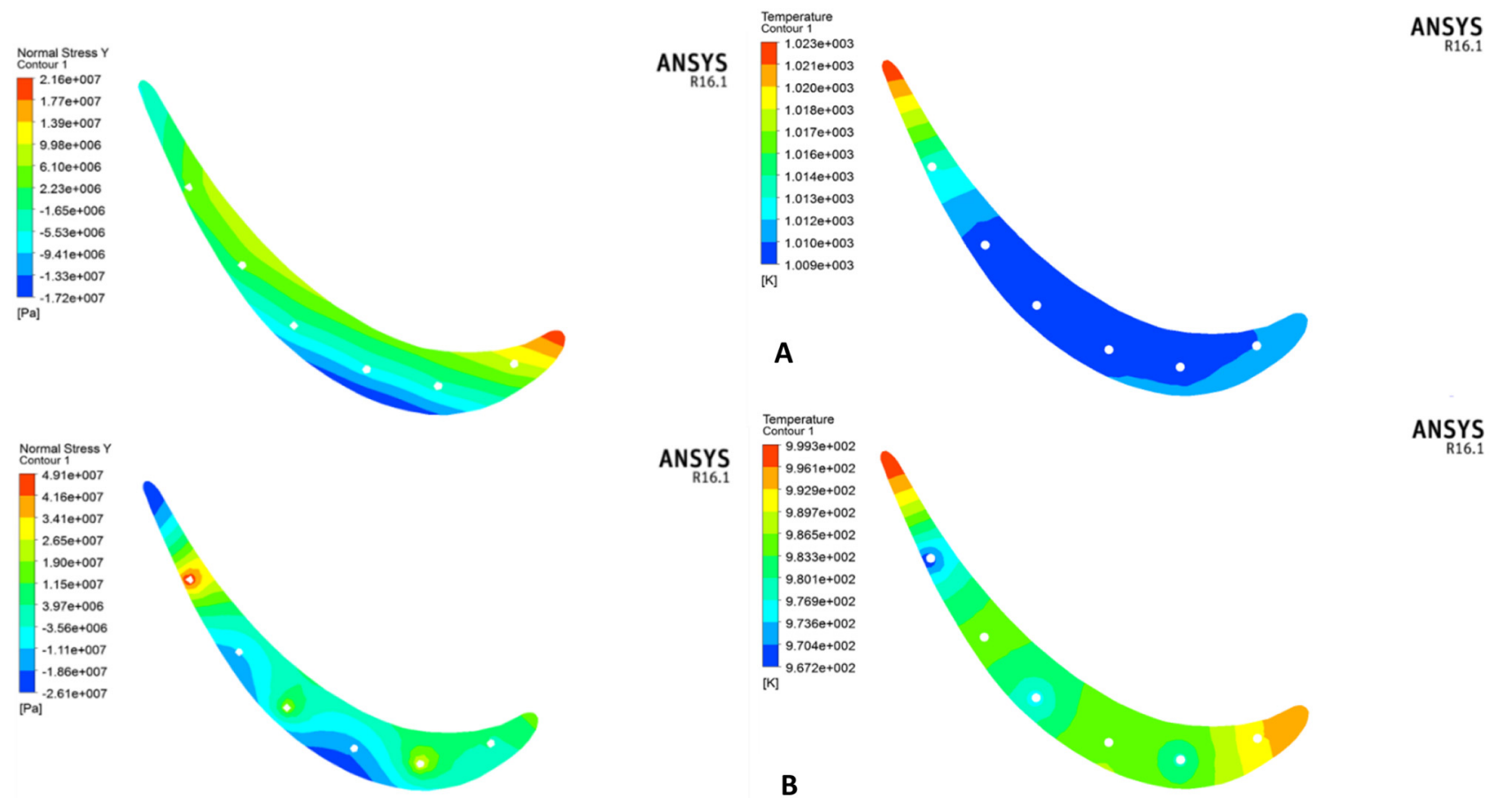

ANSYS
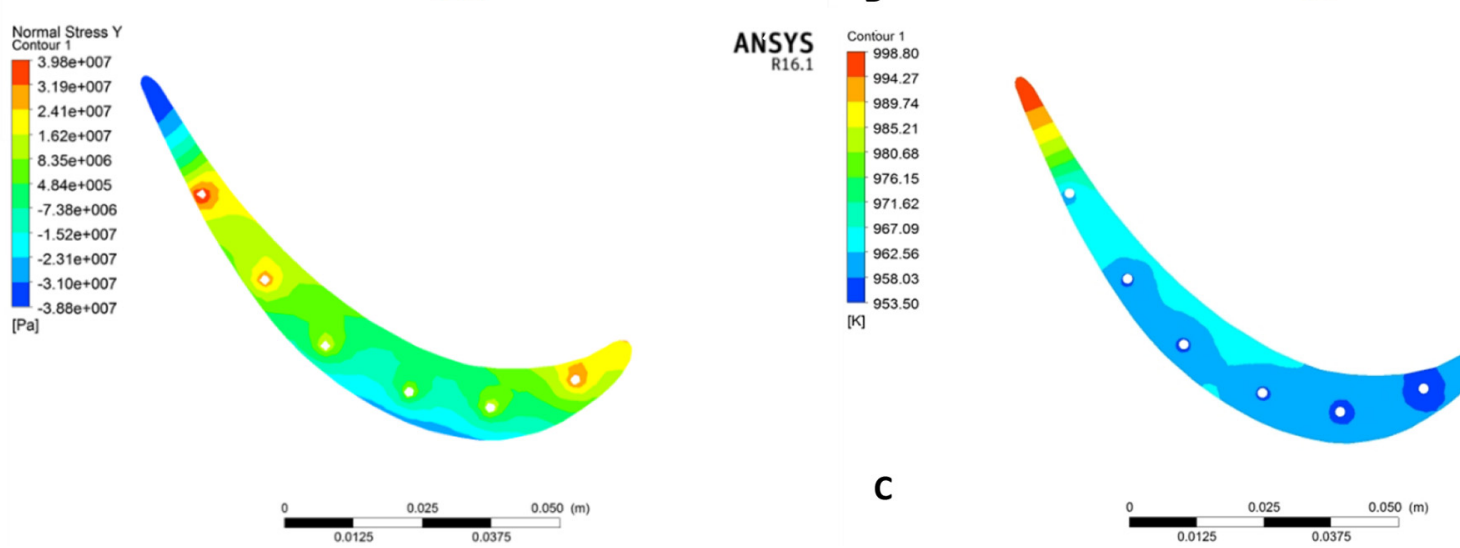

ANSYS

Fig. 12. Temperature distribution and normal stress caused by temperature gradients in section (3) at the distance of one third of the blades' length from hub of the blade in the modes (a) without cooling, (b) half-cooling, (c) full cooling.

- In this study, cooling channels was considered alternately closed, however, due to the expected weather conditions, channels would be closed consecutively which leads to create a more intense temperature gradient.

- It can be said blade in full cooling mode provides turbine blade function optimal conditions compared to the halfcooling and without cooling modes. Thanks to cooling, the blade temperature operating range is decreased and as a consequence, the material can tolerate higher stresses.

\subsection{Solutions to prevent such events}

- Some infiltration filters can be installed in the way air flow to the compressor to prevent the entrance of dusts and impurities. These filters should be visited periodically.
- Another solution is regular oversee of the hot paths of the turbine.

\section{Nomenclature}

$C_{p} \quad$ Local pressure coefficient, $\left[P_{x}-P_{\text {ref }}\right] /\left[1 / 2 p V_{b}^{2}\right]$

$C_{x} \quad$ Blade axial chord length

$P_{x} \quad$ Local pressure

$P_{\text {ref }} \quad$ Reference pressure

$F_{r} \quad$ Centrifugal force as a function of blades' radios

$S_{r} \quad$ Area of a cross section of the blade as a function of blades' radios

$R_{\text {hub }}$ Radios of the blade at hub

$R_{\text {tip }} \quad$ Radios of the blade at tip

$F_{b} \quad$ Centrifugal force of the Sherrod bonding area

$F_{b l(r)}$ Centrifugal force of a section of the blade that is between $r$ cross section and $r$ of tip of blade 

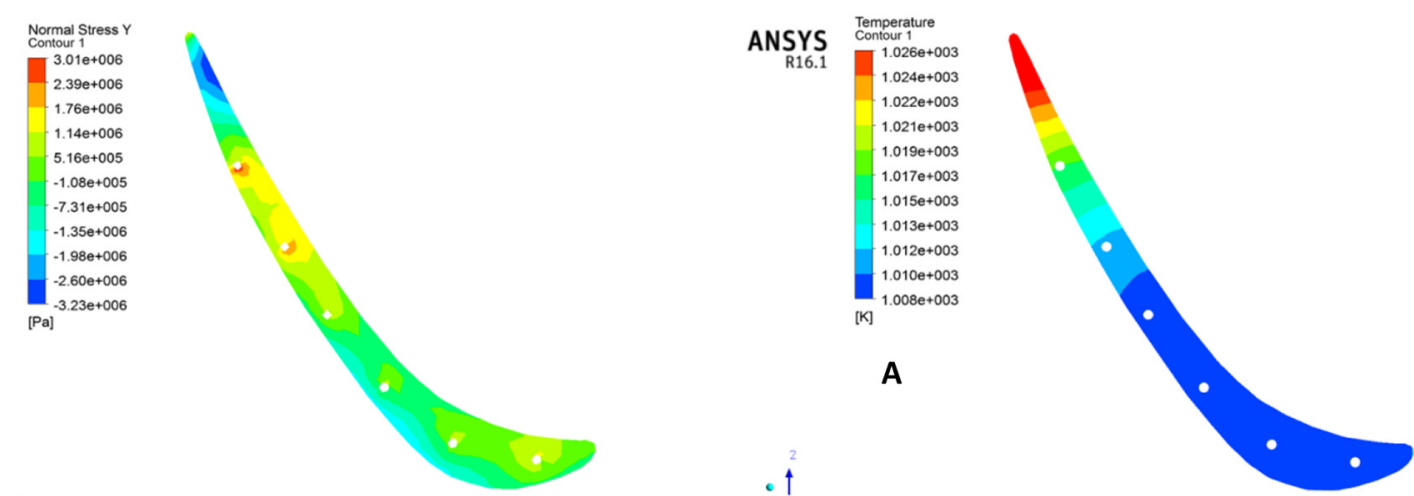

ANSYS
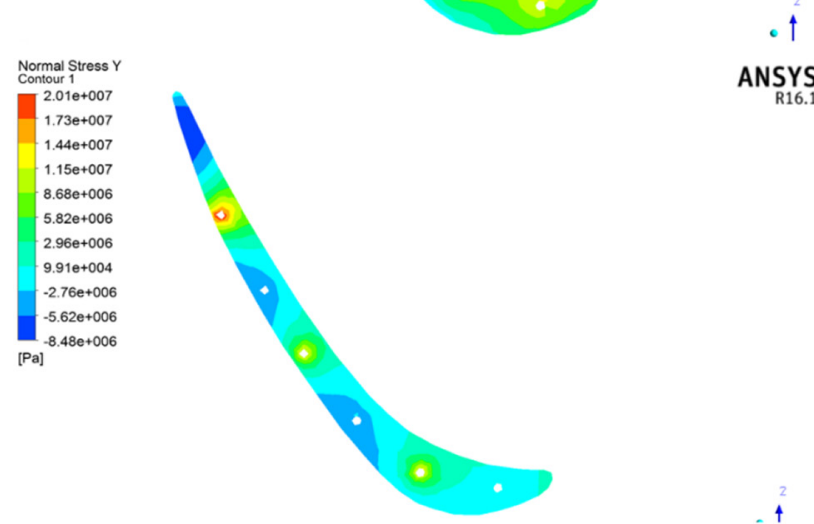

A
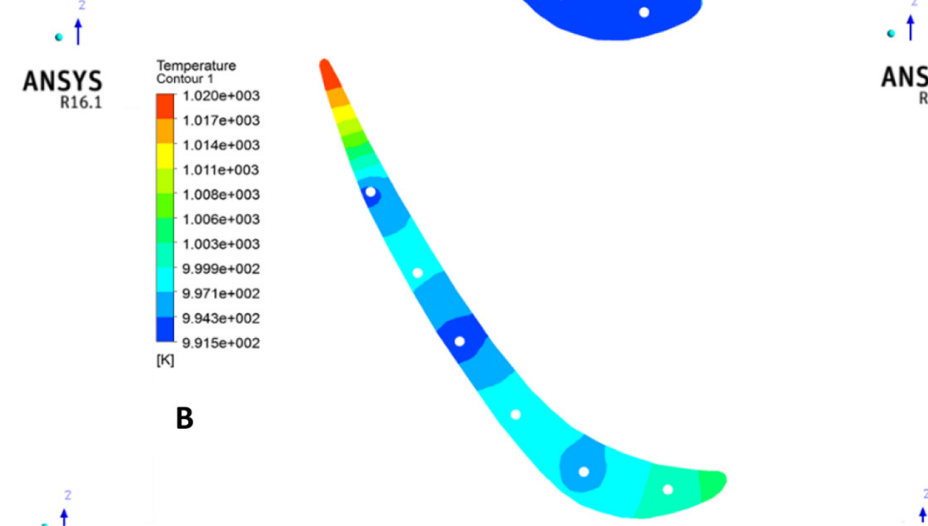

ANSYS
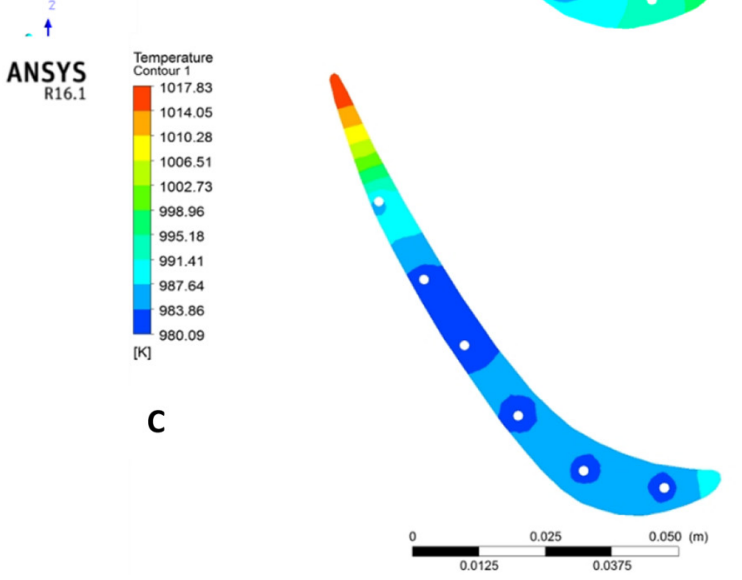

$\underset{\text { R16.1 }}{\text { ANSYS }}$

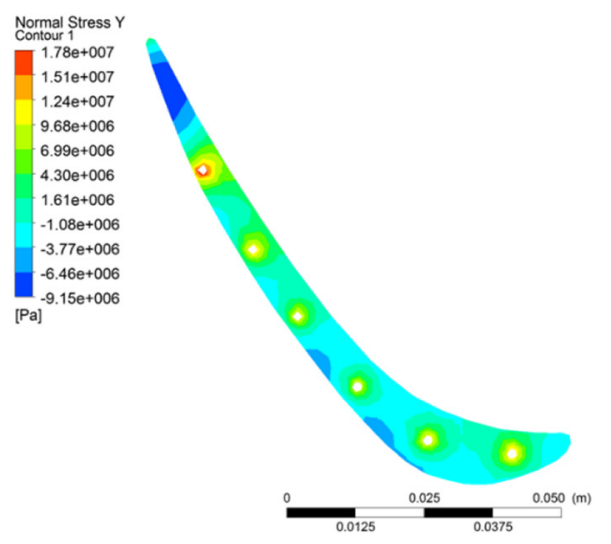

Fig. 13. Temperature distribution and normal stress caused by temperature gradients in section (4) at tip of the blade in the modes (a) without cooling, (b) half-cooling, (c) full cooling.

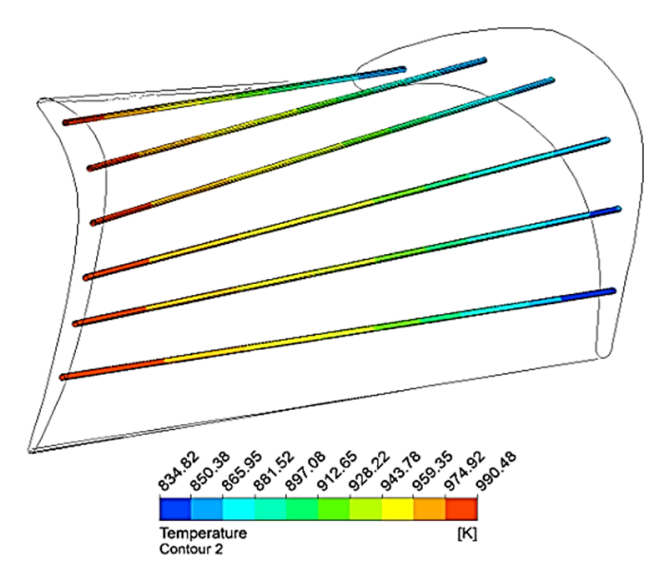

Fig. 14. Temperature distribution on the wall adjacent to the cooling fluid with 6 cooling channels.
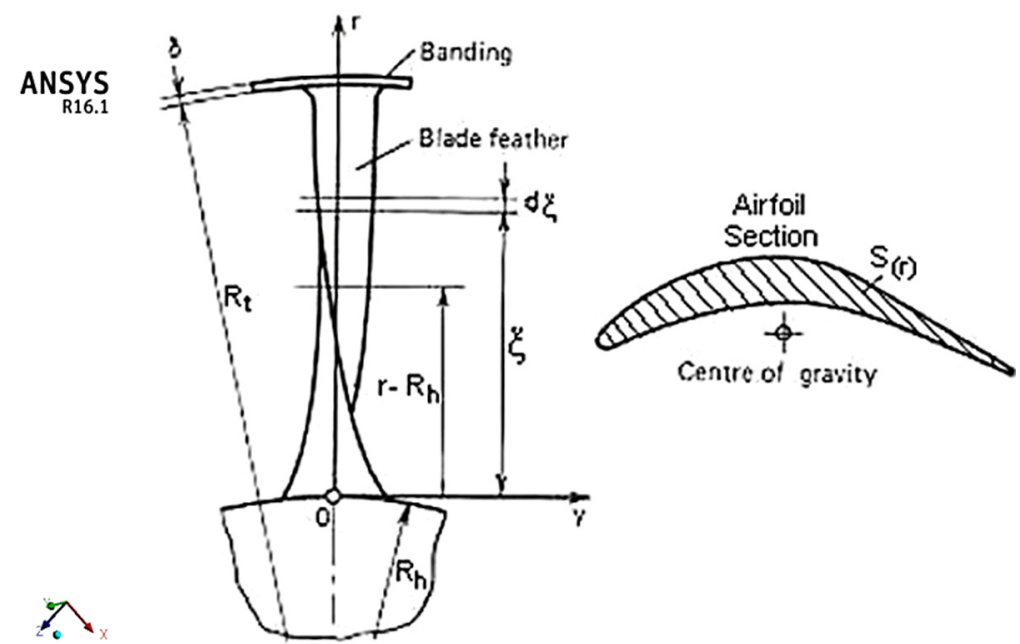

Fig. 15. Schema of a moving blade having Sherrod bonding with corresponding coordinates [16]. 


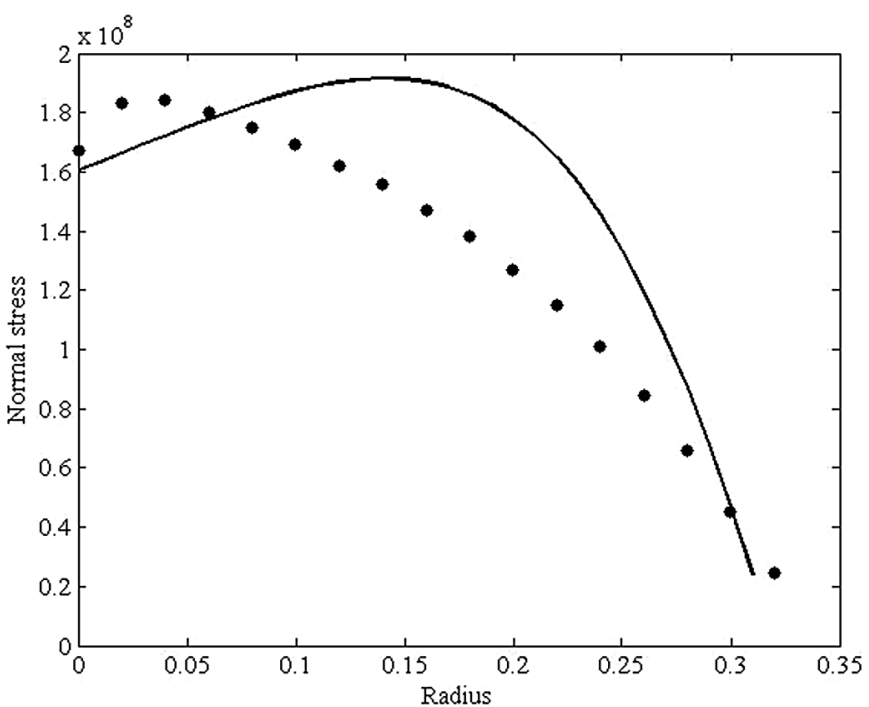

Fig. 16. The normal stress from centrifugal force.
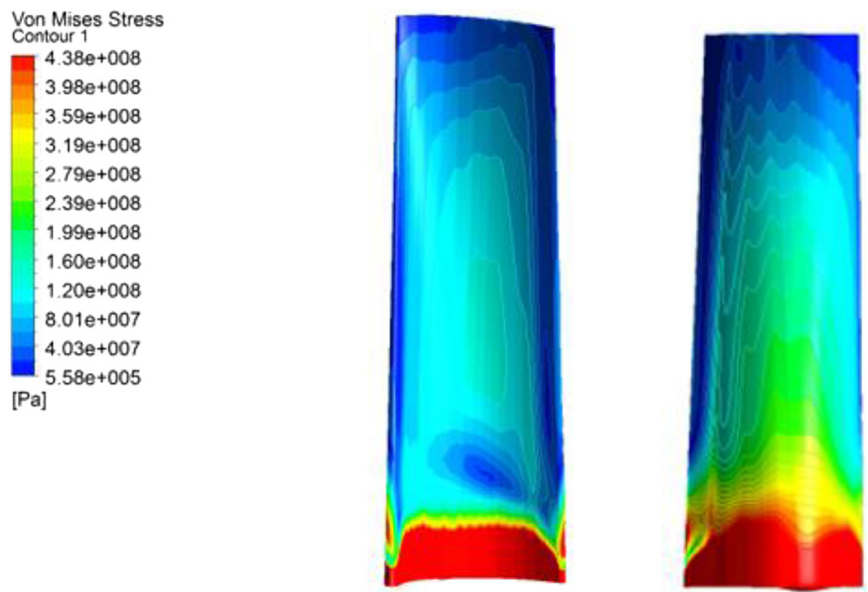

Fig. 17. Overall stress that applied on the blades in half cooling state.

\section{References}

[1] J. Polezhaev, The transpiration cooling for blades of high temperatures gas turbine, Energy Convers. Manag. 38, 1123-1133 (1997)

[2] R.M.A. El-maksoud, Gas turbine with heating during the expansion in the stator blades, Energy Convers. Manag. 78, 219-224 (2014)
[3] K.M. Kim, J.S. Park, D.H. Lee, T.W. Lee, H.H. Cho, Analysis of conjugated heat transfer, stress and failure in a gas turbine blade with circular cooling passages, Eng. Fail. Anal. 18, 1212-1222 (2011)

[4] L. Zhang, J.-C. Han, Influence of mainstream turbulence on heat transfer coefficients from a gas turbine blade, J. Heat Transfer 116, 896-903 (1994)

[5] T. Arts, M. Lambertderouvroit, A.W. Rutherford, Aerothermal investigation of a highly loaded transonic linear turbine guide vane cascade. A test case for inviscid and viscous flow computations, NASA STI/Recon Tech. Rep. N 91, 23437 (1990)

[6] R.J. Boyle, C.M. Spuckler, B.L. Lucci, W.P. Camperchioli, Infrared low temperature turbine vane rough surface heat transfer measurements, in ASME Turbo Expo 2000: Power for Land, Sea, and Air (2000) p. V003T01A024-V003T01A024

[7] F.E. Ames, C. Wang, P.A. Barbot, Measurement and prediction of the influence of catalytic and dry low NOx combustor turbulence on vane surface heat transfer, in ASME Turbo Expo 2002: Power for Land, Sea, and Air (2002), pp. 969-980

[8] C. Camci, T. Arts, An experimental convective heat transfer investigation around a film-cooled gas turbine blade, J. Turbomach. 112, 497-503 (1990)

[9] P.W. Giel, R.J. Boyle, R.S. Bunker, Measurements and predictions of heat transfer on rotor blades in a transonic turbine cascade, in ASME Turbo Expo 2003, collocated with the 2003 International Joint Power Generation Conference (2003), pp. 623-638

[10] D.-H. Rhee, H.H. Cho, Effect of vane/blade relative position onheat transfer characteristics in a stationary turbine blade: Part 2. Blade surface, Int. J. Therm. Sci. 47, 1544-1554 (2008)

[11] M.D. Polanka, D.A. Hoying, M. Meininger, C.D. MacArthur, Turbine tip and shroud heat transfer and loading: Part A-Parameter effects including reynolds number, pressure ratio, and gas to metal temperature ratio, in $A S M E$ Turbo Expo 2002: Power for Land, Sea, and Air (2002), pp. 219-230

[12] J.S. Kwak, J.-C. Han, Heat transfer coefficient and filmcooling effectiveness on a gas turbine blade tip, in $A S M E$ Turbo Expo 2002: Power for Land, Sea, and Air (2002), pp. 309-318

[13] V.K. Garg, D.L. Rigby, Heat transfer on a film-cooled bladeeffect of hole physics, Int. J. Heat Fluid Flow 20, 10-25 (1999)

[14] G. Medic, P.A. Durbin, J. Turbomach. 124, 193-199 (2002)

[15] J.R. Davis, ASM specialty handbook: heat-resistant materials. ASM International (1997)

[16] A. Kostyuk, V. Frolov, Steam and gas turbines. Mir Pub. (1988)

Cite this article as: J. Rahimi, E. Poursaeidi, E. Khavasi, Stress analysis of a second stage gas turbine blade under asymmetric thermal gradient, Mechanics \& Industry 20, 607 (2019) 